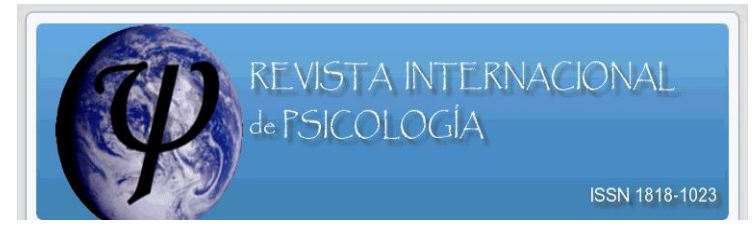

\title{
LA FAMILIA DISFUNCIONAL Y LA PSICOPATOLOGÍA EN LA POBLACIÓN DE SAN SALVADOR
}

\author{
José Ricardo Gutiérrez $\mathrm{PhD}^{1}$
}

\section{INTRODUCCIÓN GENERAL}

La presente investigación es un proyecto que se ha desarrollado con la participación de la población del área metropolitana de San Salvador; el trabajo de campo fue desarrollado desde junio a octubre del 2004, con la participación activa de veinte y dos estudiantes de la carrera de Psicología, Relaciones publicas y Administración de empresas. El trabajo de campo fue organizado en dos etapas; En la primera, se realizó la encuesta, habiéndose organizado geográficamente al área metropolitana de San Salvador en siete sectores, siendo los siguientes: Parque San José, Parque Morazán, Parque Libertad, Plaza Barrios, zona del Hula Hula, calles Rubén Darío y Arce, y por último, la UTEC.

En la segunda fase de la investigación se realizaron las entrevista televisivas a dos especialista en el tema de familia y alteraciones mentales y a un ex-pandillero. De igual forma, se hicieron diferentes visitas a sectores de San Salvador para filmar las imágenes que sustentaran el informe; posteriormente el equipo de producción continuo con el trabajo de edición del documental.

La investigación buscaba lograr los objetivos siguientes: Identificar la Prevalencia de diferentes tipos de maltrato, determinar la incidencia de Psicopatologìa en la población de San Salvador, determinar si el ambiente familiar incide en la generación de Psicopatologìa y la elaboración de un documental televisivo que evidencie la importancia de la conservación de la salud mental de los salvadoreños.

El tipo de estudio es descriptivo y correlacional, su diseño de investigación es retrospectivo, transaccional descriptivo y correlacional, ya que se midió en la población las experiencias de maltrato en la infancia, el estado actual de salud y se establecieron

\footnotetext{
${ }^{1}$ Universidad Tecnológica de El Salvador Correo electrónico: jgutierrezquintanilla@yahoo.com
} 
algunas correlaciones entre maltrato en la niñez y la presencia de psicopatologías en la vida adulta de la población. Así mismo, la técnica utilizada fue la encuesta dirigida; La muestra fue intencionada en 452 personas de ambos sexos, debido a que la encuesta estaba conformada por dos instrumentos que constituían un número de items bastante significativos y que las personas abordadas debían encontrarse estacionadas o laborando; quienes deberían de disponer del tiempo necesario para responder la encuesta. Los instrumentos utilizados son la CATS-R, que es una escala que mide abuso y trauma en la niñez y el GHQ-28, que es un cuestionario que evalúa el estado de salud en general de la población.

Con la finalidad de contextualizar a nuestros lectores se hace un breve análisis sociodemográfico de la población en estudio.

En cuanto a la edad de la muestra encuestada se encontró que más del 59\% poseen edades entre los 18 y 27 años, lo que significa que se trata de una población de jóvenes; el resto de la población estudiada tienen más de 28 años. Lo anterior nos indica que se trata de una población económicamente activa. Así mismo, se encontró que un 50.4\% de la población son del sexo femenino y un $49.6 \%$ del sexo masculino, coincidiendo perfectamente bien con las estadísticas nacionales en relación al sexo de los salvadoreños.

En relación al tipo de trabajo que desempeña la población estudiada, se encontró que un $21.2 \%$ son empleados como: secretarias, cobradores, vigilantes, un $17 \%$ son vendedores o comerciantes(no propietarios), un $16.2 \%$ expresaron ser comerciantes con negocio propio; los porcentajes anteriores suman un $54.4 \%$ de la población en estudio que se dedican a actividades comerciales, mientras el resto se dedica a diversas actividades como: Mecánica, albañilería, carpintería, zapatería, estudiantes y también se encontró un $11.5 \%$ de desempleados.

Por otra parte, en relación al estado civil de la población en estudio se encontró que un $59.1 \%$ son solteros, un $23.7 \%$ son casados, un $12.2 \%$ acompañados y un $4.6 \%$ expreso que eran divorciados. Estos datos nos indican que más de la mitad de la población estudiada son solteras y que arriba de una tercera parte de ellos han conformado un hogar. En cuanto al número de miembros en su familia se encontró que un 54.4\% de los 
entrevistados expreso que en su casa viven de una a cuatro personas, mientras un $41.6 \%$ manifiesta que en su hogar viven entre cinco y ocho personas y un $3.4 \%$ expreso que en su casa viven más de nueve personas. Los últimos datos reflejan que a pesar que existen programas de educación sexual, planificación familiar, las familias salvadoreñas siguen siendo numerosas.

En cuanto al nivel educativo alcanzado por la población evaluada se encontró que arriba de una tercera parte $(36.7 \%)$ han realizados estudios superiores, mientras un $31.9 \%$ poseen educación segundaria, un $16.2 \%$ tienen educación básica y el resto ha estudiado entre el primer y segundo ciclo. Significando que la población evaluada en su mayoría poseen una educación alta en comparación con el promedios de educación recibida por los salvadoreños que es de $5^{\circ}$. Grado. Con respecto al lugar de residencia de la población en estudio se encontró que un $83.4 \%$ residen en áreas urbanas, mientras el $14.8 \%$ viven en áreas rurales; indicando que se esta trabajando con un población que reside en la ciudad.

\section{DISCUSIÓN DE LOS RESULTADOS.}

La discusión de los resultados se ha desarrollado por áreas o tipos de maltrato de que han sido víctimas la población en estudio. También se hace una descripción de la Psicopatologìa encontrada en la población; la cual puede ser el resultado de la violencia en el hogar. A continuación se describen los hallazgos más significativos encontrados en la investigación.

Cuando se evalúa la variable resumen "abuso físico" en la población en estudio se encontró que un $41.4 \%$ expreso que nunca ha sido abusado, un 50.2\% manifestó que algunas veces, mientras un $4.0 \%$ dijo que muy a menudo y frecuentemente fue víctima de abuso físico, sólo un $4.4 \%$ no respondieron a las preguntas. Estos resultados reflejan que más de la mitad de la población investigada, cuando eran niños fueron agredidos físicamente con castigos severos; teles como: golpes con chilillos u otros objetos que les dejaban huellas, en algunos casos les dejaron moretes o heridas. Así mismo, en muchas ocasiones tuvieron que cuidarse asimismo antes que tuvieran la edad suficiente. En 
algunas ocasiones fueron golpeados en la cabeza o en la cara, sin recibir explicación alguna.

El ambiente de maltrato físico en que creció esta población puede haber jugado un factor desencadenante de diversas psicopatologías como: depresión, ansiedad y agresividad; En esta misma dirección el Dr. Gibbons, Mónico y de Arévalo(2000) plantearon que el abuso físico en la niñez es una variable que incide en las características Psicopatológicas que prevalecen en la vida adulta y que estas se expresan como: Baja autoestima, agresividad, conflictos de relaciones interpersonales, depresión y ansiedad. Por otra parte, Hall (1991), sugiere que varios rasgos estables de la personalidad pueden predisponer a la violencia. Las experiencias de violencia cuando niño, la falta de relaciones seguras y otros factores negativos durante la niñez, pueden perjudicar el desarrollo emocional haciéndolo más hostil y agresivo, en general, más impulsivo con menos autocontrol afectivo y con menos habilidades sociales.

En este mismo sentido, estudios longitudinales han demostrado (Sue David et al 1994) que en niños agresivos (con agresividad patológica) en un elevado porcentaje se convierten en antisociales y violentos y que prácticamente todos los antisociales adultos antes han tenido trastornos de conducta en su niñez y en su adolescencia.

En relación a la negligencia emocional se encontró que un $77.7 \%$ expreso que algunas veces fueron víctimas de éste tipo de abuso, un $15.3 \%$ dijo que siempre o muy a menudo fue abusado, un $1.7 \%$ manifestó que nunca y un $5.3 \%$ no respondió. Estos resultados indican que una amplia mayoría de la población estudiada, ha sido víctima por parte de sus padres o responsables de su crianza de negligencia emocional; lo cual significa qué: Cuando estaban enfermos sus padres no les reconfortaban y cuidaban; Ellos sentían que eran emocionalmente descuidados por alguno de sus padres. Así mismo, sus padres no se preocupaban por asistir a las reuniones de su escuela; cuando niño en muchas ocasiones fue dejado sólo sin el cuidado de un adulto. Por otra parte, sus padres no se esforzaban para que tuviera suficientes y buena comida, ropa adecuada y limpia; De igual forma, no se acordaban de celebrar sus cumpleaños. 
La relación y el cuidado de los padres hacia los hijos, es importante en el desarrollo de la personalidad, los niños cuyos padres los rechazan, descuidan o maltratan suelen presentar signos de perturbaciones emocionales. (Ritter, 1974, L. Yarrow, 1961). Según la Dra. Castro(2002) los niños maltratados corren un riesgo mayor de sufrir de varias afecciones, como depresión, baja autoestima, problemas de control de los impulsos, trastornos alimentarios, abuso de sustancias, conducta antisocial y trastornos del aprendizaje.

En cuanto a la antipatía ejercida por los padres hacia sus hijos, el estudio revela que un $62.1 \%$ de los encuestados dijo que nunca la ha sufrido, un $21.9 \%$ expreso que algunas veces, un $2.7 \%$ manifestó que muy a menudo o siempre fue víctima de negligencia y un $13.3 \%$ no respondió. Los resultados indican que cerca de un $25 \%$ de la población estudiada ha sido víctima de antipatía por parte de sus padres. Lo anterior significa que fueron ridiculizados por alguno de sus padres, también fue insultado y lo llamaban con sobre nombres; a veces sus padres le decían que no lo querían, sus padres fueron difíciles de complacer en cuanto a su conducta. Así mismo, constantemente fue atacado verbalmente sin razón alguna, era culpado por cosas que no hizo; De igual forma, fue maltratado físicamente cuando eran niños y adolescente, había en sus padres una actitud de preferencia hacia sus hermanos.

En consonancia con los hallazgos anteriores, Erick Erikson, citado por Sue David et al (1994), también enfatiza en las relaciones padres /hijos, señalando que el amor y la atención paternos son importantes para que el niño desarrollo una sensación de confianza en el medio social. Sin esta confianza, es probable que veamos como peligrosos, hostil y amenazados el ambiente. Como resultado pueden huir de las relaciones personales y evitar las relaciones sociales.

En relación a la disciplina excesiva, el estudio refleja que un $61.9 \%$ expreso que algunas veces fueron víctimas de disciplina excesivas, un $28.6 \%$ dijo que muy a menudo o siempre, un $3.7 \%$ manifestó que nunca y un 5.8\% no respondió. Indicando que más del 90\% de la población investigada fue víctima por parte de sus padres de una disciplina excesiva; Consistiendo estas en que los padres exigían que siguieran reglas estrictas de conducta en el hogar y cuando eran castigados severamente no les explicaban los motivos del castigo. También sus padres fueron estrictos con las horas de llegada a 
casa; Expresan que el grado de castigo que recibían nunca fue apropiado. De igual forma, sus padres les imponían reglas estrictas de conducta, tales como: de cortesía e higiene.

En relación a la discordia y tensión en el hogar en el estudio se encontró que un 33\% han sido víctimas algunas veces de discordia, un $4 \%$ refiere que muy a menudo o siempre fue víctima de este maltrato, un $58.2 \%$ dijo que nunca, un $4.8 \%$ no respondió. Los datos anteriores reflejan que más de una tercera partes de la población investigada, ha sufrido discordia y tensión en el hogar; Significando que cuando fueron niños o adolescentes observaron peleas físicas entre sus padres, vivenciado que sus padres se ofendían uno al otro y que eran infelices entre sí, Por otra parte, fueron testigos de que sus padres se tiraban objetos y quebraban cosas cuando discutían; También expresan que sus padres bebían fuertemente o usaban drogas. En este sentido, Ritchers y Martínez, (2002) informaron que los niños que presenciaban violencia, tanto en el hogar como en la comunidad tenían altos índices de estrés y los elementos específicos de distrés psicológico en niños expuestos a violencia incluyen: depresión, ansiedad, disociación, y estrés postraumático.

También Lai (2002) encontró mayores niveles de depresión y menor autoestima en adolescentes con alta exposición a violencia, ya sea que se trate tanto de testigo o como víctima de la misma. Mientras tanto, Schwab-Stone y colaboradores hallaron que la exposición a la violencia estaba estrechamente asociada con síntomas de externalización e internalización durante la adolescencia. Los síntomas de internalización (ansiedad, depresión y somatización) eran más frecuentes en los jóvenes adolescentes, en comparación con los adolescentes mayores. De igual forma, se encontró relación entre la exposición a la violencia y un menor desempeño académico, así como éste último también se asoció a una baja autoestima. En definitiva, la coexistencia de baja autoestima, pobre desempeño académico y la exposición a la violencia, hace a estos niños particularmente vulnerables a los comportamientos de riesgo, incluyendo el comportamiento violento.

En cuanto al abuso sexual en la población se encontró que un $10.3 \%$ expreso que algunas veces ha sido víctima de este maltrato, un $0.5 \%$ manifestó que muy a menudo o siempre, un $77 \%$ dijo que nunca, un $12.2 \%$ no respondió. Estos datos indican que sólo 
una pequeña proporción de la muestra estudiada ha sido abusada sexualmente; lo cual significa qué, antes que tuviera los diez y seis años una persona mayor( 5 años) lo manoseo sexualmente, que fue víctima de manoseo sexual por otro joven sin que él / ella lo quisiera. También se refiere a que cuando eran niños o adolescentes tuvo relaciones sexuales traumáticas; De igual forma, tuvieron relaciones sexuales no deseadas con otro / otra joven por que él / ella uso amenazas o fuerza física.

Es importante destacar que los porcentajes antes mencionados no coinciden con los resultados obtenidos en otras investigaciones realizadas en El Salvador; En el año 2000, por el Dr. Gibbons et al, quien investigo a una población de estudiantes universitarios y encontró que el abuso sexual global, es decir, el total entre hombres y mujeres víctimas de abuso sexual fue de $38 \%$. Un factor importante de mencionar es que en ese estudio, se utilizó la encuesta colectiva,(anónima) lo cual significa, la no identificación del encuestado, favoreciendo la veracidad y validez de las respuestas de los encuestados; Mientras en la investigación actual, se utilizo la encuesta dirigidas, es decir, en encuestador tenía que leer las preguntas al encuestado, lo que producía inseguridad, temor y desconfianza en el encuestado. En resumen, se puede afirmar que las diferencias en cuanto a las incidencias de ambos estudios, se deben a las características propias del estudio, a la metodología utilizada y al tipo de población que fue dirigida la investigación.

\section{Psicopatologías encontradas}

En relación al consumo de drogas o alcohol, se les pregunto a la población, si algunos de sus amigos o compañeros de trabajo consumen algún tipo de sustancias psicoactivas y un $34.3 \%$ manifestó que de vez en cuando la consumen, un $8.3 \%$ dijo que muy a menudo, un $6.4 \%$ expreso que siempre consumen drogas o alcohol y un $50 \%$ dijo que nunca. Lo anterior significa que cerca de un cincuenta (48.9\%)por ciento de la población estudiada consume algún tipo de sustancia psicoactiva. Estos resultados indican que probablemente el consumo de drogas y alcohol sea un factor importante en la generación de múltiples alteraciones, tanto de carácter social, como orgánicas y psicológicas en la población investigada. 
En lo social, se pueden mencionar: inasistencia laboral, conflictos interpersonales (peleas), violencia intrafamiliar y problemas económicos; En lo orgánico, se encuentran afecciones como: Gastritis, ulceras gástricas y duodenales, problemas hepáticos, cirrosis y anemias, entre otras. En lo Psicológico, se presentan: Irritabilidad, agresividad, pobre control de impulsos, mal humor, depresión, ansiedad y conflictos de relaciones interpersonales.

Por otra parte, al preguntar directamente a esta misma población, ¿Sí usted consume algún tipo de sustancia psicoactiva (alcohol y drogas)? se encontró que un $23.9 \%$ manifestó que sí. De éstos un $8.8 \%$ son del sexo femenino y $15 \%$ del sexo masculino. Indicando que hay una mayor Prevalencia del consumo en hombres en comparación con las mujeres. También se observa claramente una diferencia muy marcada en reconocer que sus amigos y compañeros de trabajo consumen alguna sustancia psicoactiva y aceptar que ellos consumen este tipo de sustancias, es decir, es más fácil expresar que las personas que conozco consumen drogas y alcohol, que aceptar que yo consumo algún tipo de sustancia psicoactiva.

Cuando evaluamos la Psicopatologìa en la población de San Salvador se encontró que un $19.6 \%$ de casos de disfunción social, alteración que se manifiesta por una incapacidad para mantenerse ocupado y activo, les cuesta mucho hacer las cosas rutinarias, presentan la sensación general de que no esta haciendo las cosas bien; existe una insatisfacción con la manera de hacer las cosas, también sienten que no están jugando un papel valioso en su vida y presentan un sentido de incapacidad para tomar decisiones. De igual forma, se encontró un $16.0 \%$ de casos de somatización; lo cual significa que éste segmento de la población estudiada no se siente perfectamente bien de salud y en plena forma, sienten la necesidad de un reconstituyente, existe la sensación de agotamiento y sin fuerzas para hacer nada, hay dolores de cabeza permanentes. Estas personas presentan oleadas de calor o escalofrió y un sentido de presión en la cabeza o siente que les va ha estallar.

Por otra parte, en el estudio se encontró un $13.8 \%$ de casos de ansiedad e insomnios, éste trastorno indica que la población presenta mucha preocupación que le ha hecho perder mucho sueño, es decir, no han podido dormir sin interrupciones durante la noche, se han sentido constantemente agobiados y tensos; también se sienten con los nervios a 
flor de piel y malhumorados, con sensación de pánico sin motivo alguno, han tenido la impresión de que todo le viene en cima.

Según Rodrigo P. Et al(2003) La permanencia del trastorno de ansiedad registrado en los animales aislados, a pesar de haberles restituido su entorno social habitual, indica que esta experiencia temprana deja una secuela conductual caracterizada como un "rasgo" más que un "estado" de ansiedad (Ashman et al 2002). Es decir, es una condición que perdura a pesar de que su causa originaria (aislamiento social) ya no esté presente. Así, nuestros resultados conductuales contribuyen a reafirmar el hecho clínicamente bien establecido de que las experiencias tempranas hostiles dejan secuelas conductuales que, no obstante seguir un curso incierto, incrementan las probabilidades de que el niño presente una o más psicopatologías del desarrollo (Lipman et al 2001, Macmillan et al 2001, Bowlby 1988, Rutter et al 1999).

Es importante destacar que según las estadísticas de atención primaria en salud que el Ministerio de Salud Pública y Asistencia Social (2003), ha documentado en los últimos años. Resulta extremadamente alarmante que el trastorno de ansiedad ocupe el primer motivo de consulta en los últimos años.

En relación a la depresión se encontró solamente un $6.4 \%$ de casos en la población con esta alteración mental. Lo anterior significa que existe un grupo de personas que piensan que no sirven para nada (sentimiento de inutilidad), que viven la vida sin esperanza, tienen la sensación de que la vida no merece la pena vivirla, también han pensado en la posibilidad de quitarse la vida y que desean estar muertos y lejos de todo; esta idea de quitarse la vida viene repetidamente a la cabeza.

Por otra parte, es importante destacar que cada uno de los casos de Psicopatologìa antes mencionados son excluyentes, es decir, que al sumar estos porcentajes, se observa que un $55.8 \%$ de la población estudiadas adolece de alguna de las psicopatologías ya mencionadas; Esto sin incluir el consumo de drogas y alcohol.

En esta misma dirección, un estudio realizado por Gutiérrez et al(2002) con la población de menores infractores internados a nivel nacional, determinó que un $65.5 \%$ de los jóvenes presentan alteraciones significativas a su salud mental, entre los trastornos 
encontrados se pueden mencionar: Hopocondriasis, desviación psicopática, trastorno paranoide, esquizofrenia e hipomanía. También el 100\% de los menores infractores en reeducación consumen alcohol o drogas. El informe concluye afirmando qué "tanto las condiciones del ambiente familiar disfuncional, como el consumo de drogas y alcohol, son factores que inciden en la salud mental de los jóvenes infractores en El Salvador.

Es importante mencionar que recientemente fue publicada (2004) la última investigación titulada "Diagnostico de Salud Mental de la Población de El Salvador", realizada por Gutiérrez (2003)" con una muestra de 1,668 personas seleccionadas aleatoriamente a nivel nacional, y esta refleja que un $36 \%$ de los salvadoreños presentan síntomas de trastornos mentales y del comportamiento, tales como: Obsesividad, angustia, ansiedad, somatización, depresión, Síndrome convulsivo, Síndrome cerebral orgánico, psicosis, disociación y conversión, neurosis y alcoholismo; Sin embargo, en el estudio no se logro determinar los factores o variables causales responsables de la generación de los síntomas de Psicopatologìa.

Al contrastar los resultados de Psicopatologìa en los estudios antes mencionados, se puede observar que existen diferencias muy sutiles, en el sentido que si comparamos los hallazgos en los menores infractores con los resultados de la investigación actual, observamos que las diferencia es menor del $10 \%$ en contra de los menores infractores, es decir, existe un $9.7 \%$ mayor de casos en los menores infractores que en la población del área metropolitana de san salvador. Sin embargo, hay que destacar que la gravedad de los trastornos o el nivel de deterioro psíquico y de la personalidad de los menores infractores es más delicado.

Cuando se comparan los resultados de la investigación actual con la realizada el año 3003 a nivel nacional, encontramos que en la primera se ha hecho una sumatoria de los porcentajes de cada trastornos, mientras en el estudio del 2003, son un promedio que resulto de haber sumados todos lo porcentajes(de diferentes trastornos) y divididos entre los diez trastornos estudiados, lo cual indica que existen varios casos, donde sus porcentajes superan el $40 \%$ de prevalencia de síntomas de psicopatologías.

Lo anterior significa que la investigación a nivel nacional refleja mayor prevalencia de Psicopatologìa que la realizada en el área metropolitana de San Salvador; hay que 
mencionar que esta investigación es mas representativa de la población salvadoreña por haberse encuestado una muestras de 33 municipios del país. También podría suceder que las metodologías utilizadas en cada investigación este incidiendo en los resultados de cada estudio.

\section{CONCLUSIONES}

Según el análisis de los resultados, más del cincuenta por ciento de la población investigada fue víctima de maltrato físico durante la niñez y la adolescencia, a quienes les aplicaban castigos severos, tales como: golpes con chilillos u otros objetos que les dejaban huellas, como moretes y heridas, tanto en el cuerpo como en cara y la cabeza.

Los resultados indican que más del noventa por ciento de la población estudiada, sufrió negligencia emocional cuando eran niños o adolescentes, por parte de sus padres o los responsables de su crianza; la negligencia emocional, fue expresada en acciones y omisiones, tales como: los padres no se preocupaban de reconfortarlo cuando estaba enfermo o triste, no les procuraban buena alimentación y vestuario limpio, tampoco se preocupaban por el rendimiento académico y las actividades de la escuela y muchas veces fueron dejados solos, sin el cuidado de un adulto.

El análisis de los hallazgos evidencian que una de cada cuatro personas de la población investigada fue víctima de antipatía en su niñez y adolescencia, por parte de sus padres; la cual consistía en que eran ridiculizados por alguno de sus padres, fueron insultados o llamados con sobrenombres, sus padres frecuentemente les decían que no los querían, eran atacados verbalmente sin razón alguna; también fueron culpados por cosas que no hicieron.

El análisis refleja que más del noventa por ciento de la población investigada ha sido víctima de disciplina excesiva, cuando fueron niños o adolescentes por parte de sus padres, consistiendo esta en que los padres exigían reglas estrictas de conducta en el hogar; Cuando eran castigados no les explicaban los motivos del castigo, también fueron estrictos con las horas de llegada a casa. De igual forma, el grado de castigo nunca fue apropiado, es decir, que el castigo no era proporcional a la falta cometida. La disciplina excesiva en muchas veces esta relacionada con el abuso físico y 
psicológico; siendo la disciplina en muchas ocasiones una forma disfrazada de otros tipos de abuso que coexisten en el hogar de los salvadoreños.

El estudio nos indica que más de una tercera parte de la población investigada sufrió de discordia y tensión en el hogar, cuando eran niños y adolescentes; Esto significa que cuando fueron niños observaron peleas físicas entre sus padres, experimentando que sus padres se ofendían uno al otro y que eran infelices entre sí; también fueron testigos de que sus padres se tiraban objetos y quebraban cosas cuando discutían y que sus padres bebían fuertemente y consumían drogas.

Los resultados indican que cerca del cincuenta(48.9\%) por ciento de la población estudiada consume algún tipos de sustancia psicoactiva y que estas pueden ser alcohol o drogas. Estos hallazgos probablemente son un factor importante en la generación de múltiples alteraciones de carácter orgánico, psicológico y social en la población investigada.

Al evaluar la Psicopatologìa en la población estudiada, se encontró que más del cincuenta por ciento(55.8\%)presentan alguna alteración o síntomas de trastornos mentales, como: Ansiedad, depresión, somatización y disfunciones sociales; Las cuales han sido influenciadas o determinadas por las condiciones del ambiente familiar disfuncional en el que se desarrollaron cuando eran niños o adolescentes.

El análisis inferencial demostró que aquellas personas que fueron víctimas de abuso físico por parte de sus padres cuando eran niños o adolescentes, en la adultez presentan síntomas de alteraciones o trastornos mentales como: Somatización, disfunción social y ansiedad; Por otra parte, la negligencia emocional es un factor que incide o propicia la generación de las psicopatologías antes mencionadas. En este mismo sentido, el análisis inferencial mediante la prueba " $t$ " de student evidencia que los niños y adolescentes que fueron víctimas de una disciplina excesiva presentan síntomas de alteraciones mentales como: Disfunción social, somatización y ansiedad. También la discordia y tensión en el hogar es una variable que incide en las psicopatologías antes mencionadas. 
La prueba " $\mathrm{t}$ " de student, ha demostrado que el consumo de drogas y alcohol en la población investigada, ha afectado significativamente el funcionamiento social de la población; De igual forma, este comportamiento desadaptado incide en la generación de síntomas de los trastornos de somatización, ansiedad e insomnio y depresión.

\section{RECOMENDACIONES}

El Estado, mediante el Ministerio de Educación y el Ministerio de Salud Publica y Asistencia Social, diseñen y administren proyectos y programas de prevención y educación, sobre las formas adecuadas de criar a los hijos. Estos programas deberán ser administrados y orientados, hacia los niños, los jóvenes y padres de familia. Contribuyendo de esta manera en la disminución o eliminación de los diferentes tipos de maltrato de que son víctimas los niños y los adolescentes salvadoreños. También de esta forma, se estará previniendo las incidencias de psicopatologías que en la actualidad presenta la población investigada.

Las instituciones de educación superior, incorporen en sus programas de estudio, signaturas, áreas didácticas o contenidos temáticos sobre estilos de crianza, modelos de padres, efectos de la violencia familiar, tipos de maltrato, derechos humanos, equidad de genero, entre otros, con la finalidad de minimizar el impacto que en la actualidad están sucediendo en el ambiente familiar. Es decir, que se contribuyan con la formación y educación de los futuros padres, sobre las formas adecuadas de organizar, dirigir y establecer las relaciones entre los miembros de la familia.

Las instituciones oficiales autónomas, fundaciones, ONGs, desarrollen y fortalezcan los proyectos y programas educativos orientados hacia la convivencia saludable de la familia, debido a que en el seno de esta, se configuran y se definen diversas psicopatologías que afectan al individuo y la sociedad salvadoreña.

Que el Ministerio de Salud Pública y Asistencia Social y las instituciones estatales y privadas vinculadas con la salud de la población, diseñen estrategias, proyectos y programas masivos orientados a la prevención y educación de los efectos físicos, psicológicos y sociales del consumo de drogas y alcohol en la sociedad salvadoreña. 
El Programa Nacional de Salud Mental(MSPAS)diseñe y implemente programas de promoción, educación, prevención y curación, orientados a aquellas alteraciones mentales más prevalecientes en la sociedad salvadoreña. Entre los trastornos mas frecuentes se tiene: La ansiedad, depresión, somatización, entre otras. Programas que pueden ser de alcance nacional, regional, departamental o local.

Los profesionales de salud mental incentiven, promuevan y apliquen programas preventivos, curativos y de rehabilitación de los trastornos mentales y del comportamiento. Trabajo que puede ser ejercido desde el quehacer profesional privado e institucional, donde desarrollan su labor profesional, contribuyendo es esta manera en la conservación de la salud mental de los salvadoreños.

\section{REFERENCIAS.}

1. Ashman SB, Dawson G, Panagiotides H, Yamada E, Wilkinson CW.(2002) Stress hormone levels of children of depressed mothers. Dev Psychopathol 2002; 14: 333-349 [ $\underline{\text { Medline ] }}$

2. Alcaina P. Badajoz L.(2004) Psicopatologìa e Interacción Familiar.http://www.sepsiquiatria.org/sepsiquiatria/html/informacion_sociedad/ manual/a5n11.htm.

3. Acevedo G. (2002) Pediatr Adolesc Med, americasalud.com.uy;155: 13511356. JAMA. Enero.

4. Brown GW, Monck EM, Castairs GM, et al.(1962) Influence of family life on the course of schizophrenic illness. Br J Prevent Social Med; 16: 55-68.

5. Bebbington PE and Kuipers L.(1982) The social management of schizophrenia. Br J Hosp Med; 28: 396-403.

6. Barrowclough C, Tarrier N.(1984) Psychosocial intervention with families and their effects on the course of schizophrenia: a review. Psychol Med; 14: 629642.

7. Bremner JD, Narayan M, Staib LH, Southwick SM, McGlashan T, Charney DS.(1999) Neural correlates of memories of childhood sexual abuse in women with and without posttraumatic stress disorder. Am J Psychiatry; 156: 17871795 [ Medline $]$ 
8. Bowlby J. A(1988) secure base: Clinical applications of attachment theory. Routledge, Londres.

9. Bush G, Luu O, Posner MI.(2000) Cognitive and emotional influences in anterior cingulate cortex. TICS; 4: 215-222.

10. Coryell W, Winokur G, Maser JD, et al.(1994) Recurrently situational (reactive) depression: a study of course, phenomenology and familial psychopathology. J Affect Disord; 31: 203-210.

11. De Bellis MD, Keshavan MS, Spencer S, Hall J.(2000) N-acetylaspartate concentration in the anterior cingulate of maltreated children and adolescents with PTSD. Am J Psychiatry; 157: 1175-1177.[ Medline ]

12. Dawson G, Frey K, Panagiotides H, Yamada E, Hessl D, Osterling J.(1999) Infants of depressed mothers exhibit atypical frontal electrical brain activity during interactions with mother and with a familiar, nondepressed adult. Child Dev ; 70: 1058-1066 [ $\underline{\text { Medline }] ~}$

13. Damasio AR.(1998) Emotion in the perspective of an integrated nervous system. Brain Res Rev; 26: 83-86.

14. Dawson G, Hessl D, Frey K.(1994) Social influences on early developing biological and behavioral systems related to risk for affective disorder. Dev Psychopathol; 6: 759-779.

15. Davidson RJ, Irwin W.(1999) The functional neuroanatomy of emotion and affective style. TICS ; 3: 11-21

16. Fromm-Reichman F.(1948) Notes on the development of treatment of schizophrenia by psychoanalytic psychopathology. Psychiatry, 11: 263-273.

17. Fendrich M, Warner V and Weissman MM.(1990) Family risk factors, parental depression, and psychopathology in offspring. Developmental Psychology, 1990; 26: 40-50.

18. Gutiérrez JR;(2003) Prevalencia de síntomas de alteraciones mentales de la población de El Salvador. Diagnóstico de Salud Mental(Tomo II); Editorial Tecnoimpresos, San Salvador.

19. Gutiérrez JR, Flores HC;(2003) El ambiente familiar durante la niñez, el consumo de alcohol y drogas, y su incidencia en los rasgos de Psicopatologìa de los menores infractores en El Salvador, Impresores Diseño y Color, San Salvador. 
20. Gutiérrez JR, Flores HC.(2001)Factores incidentes en el consumo de sustancias psicoactivas y la prevalencia de características psicopatológicas de los adictos en El Salvador. Informe de investigación de la Universidad Tecnológica, San Salvador

21. Gutiérrez JR, Landaverde AC;(1998) La violencia intrafamiliar como consecuencia del alcoholismo en los oficiales de mandos medios de la Fuerza Armada; Tesis para optar al grado de Licenciados en Psicología, Universidad Tecnológica de El Salvador, San Salvador.

22. Gibbons P, Flores HM, Mónico M;(2000)" Oscuridad de la casa" La realidad escondida del abuso sexual, el maltrato infantil y sus efectos psicológicos en El Salvador, Editorial Tecnoimpresos, San salvador.

23. Goldman SJ, D'Angelo EJ and DeMaso(1993). Psychopathology in the families of children and adolescents with borderline personality disorder.Am $\mathrm{J}$ Psychiatry, 150: 1832-1835.

24. Green SM, Loeber R and Lahey BB. Child psychopathology and deviant family hierarchies. Journal of Child \& Family Studies, 1992; 4: 341-349.

25. Grigoroiu-Serbanescu M, Christodorescu D, Jipescu I, et al.(1990) Children aged 10-17 of endogenous unipolar depressive parents and normal parents: I. Psychopathology rate and relations of the severity of the psychopathology to familial and environmental variables. Rom J Neurol Psychiatry; 28: 45-62.

26. Gunnar MR, Morison SJ, Chisholm K, Schuder M.(2001) Salivary cortisol levels in children adopted from Romanian orphanages. Dev Psychopathol; 13: 611-628, [ $\underline{\text { Medline }]}$

27. Grachev ID, Apkarian AV.(2000) Anxiety in healthy humans is associated with orbital frontal chemistry. Mol Psychiatry; 5: 482-488. [ $\underline{\underline{M e d l i n e}}$ ]

28. Helmeke C, Poeggel G, Braun K.(1001) Differential emotional experience induces elevated synaptic densities on basal dendrites of piramidal neurons in the anterior cingulate cortex of Octodon degus. Neuroscience; 104: 927-931 [ Medline ]

29. Helmeke C, Poeggel G, Braun K.(2001) Differential emotional experience induces elevated synaptic densities on basal dendrites of piramidal neurons in the anterior cingulate cortex of Octodon degus. Neuroscience; 104: 927-931[ Medline ] 
30. Haracz JL.(1985) Neural plasticity in schizophrenia. Schizophrenia Bull; 11: 191-229

31. Información especializada de fundación peruana,(2004) Familia funcional y disfuncional; disponible en http://www.opcionperu.org.pe/Web_OpcionPeru/Descarga/lafamilia.pdf

32. Ionescu A.(1985)Clinical phenomena of family psychopathology: Diagnostic reference points from the perspectives of family psychopathology. Revue Romaine des Sciences Sociales-Serie de Psychologie; 29: 147-156

33. Johnson JG, Cohen P, Brown J.(1999) Childhood maltreatment and increased risk for personality disorders during early adulthood. Arch Gen Psychiatry ; 56: 600-606

34. Johnson JG, Cohen P, Smailes EM, Skodol AE, Brown J, Oldham JM.(2001) Childhood verbal abuse and risk for personality disorders during adolescence and early adulthood. Comprehen Psychiatry; 42: 16-23.

35. Leverich GS, McElroy SL, Suppes T, Keck PE, Denicoff KD, Nolen WA, et al.(2002) Early physical and sexual abuse associated with an adverse course of bipolar illness. Biol Psychiatry; 51: 288-297.

36. Levitan RD, Parikh SV, Lesage AD, Hegadoren KM, Adams M, Kennedy SH, et al.(1998) Major depression in individuals with a history of childhood physical or sexual abuse: relationship to neurovegetative features, mania, and gender. Am J Psychiatry; 155: 1746-1752

37. Lindelöw M.(1999) Parent-child interaction and adult depression: a prospective study. Acta Psychiatr Scand; 100: 270-278 [ $\underline{\text { Medline }] ~}$

38. Lipman EL, MacMillan HL, Boyle MH.(2001) Childhood abuse and psychiatric disorders among single and married mothers. Am J Psychiatry; 158: 73-77 [ Medline ]

39. Lidz T, Cornelison AR, Fleck S, et al.(1957) The intrafamilial environment of schizophrenic patients: II. Marital schism and marital skew. Am J Psychiatry; 114:241-244.

40. Mckittrick CR, Magariños AM, Blanchard DC, Blanchard RJ, McEwen BS, Sakai RR.(2000) Chronic social stress reduces dendritic arbors in CA3 of hippocampus and decreases binding to serotonin transporter sites. Synapse; 36: 85-94 
41. Magariños AM, McEwen BS.(1985) Stress-induced atrophy of apical dendrites of hippocampal CA3 neurons: involvement of glucocorticoid secretion and excitatory amino acid receptors. Neuroscience; 69: 89-98

42. MacMillan HL, Fleming JE, Streiner DL, Lin E, Boyle MH, Jamieson E, et al.(2001) Childhood abuse and lifetime psychopathology in a community sample. Am J Psychiatry; 158: 1878-1883

43. Narnow JR, Thompson M, Hamilton EB, et al.(1994) Family-expressed emotion, childhood-onset depression, and childhood-onset schizophrenia spectrum disorders: is expressed emotion a nonspecific correlate of child psychopathology or a specific risk factor for depression?. J Abnorm Child Psychol; 22: 129-146.

44. Princhard JW.(1996) MRS of the brain-prospects for clinical application in MR spectroscopy. En: Clinical Applications and Techniques. Young IR, Charles HC (Eds.), London, Livery House, pp 1-25

45. Poeggel G, Lange E, Hase C, Metzger M, Gulyaeva N, Braun K.(1999) Maternal separation and early social deprivation in Octgodon degus: quantitative changes of nicotinamide adenine dinucleotide phosphatediaphorase-reactive neurons in the prefrontal cortex and nucleus accumbens. Neuroscience ; 94: 497-504[ $\underline{\text { Medline ] }}$

46. Pascual R. (2002) La interrupción temprana del vínculo social altera la organización citoarquitectónica y expresión de neuropéptidos en la corteza prefrontal. Rev Chil Neuro-Psiquiat; 40: 9-20.

47. Pascual R. (2002)La interrupción temprana del vínculo social altera la organización citoarquitectónica y expresión de neuropéptidos en la corteza prefrontal. Rev Chil Neuro-Psiquiat; 40: 9-20

48. Poeggel G, Nowicki L, Braun K.(2003) Early social deprivation alters monoaminergic afferents in the orbital prefrontal cortex of Octodon degus. Neuroscience; 116: 617-620

49. Rodrigo P, Catalán M, Fuentealba M.(2003) Revista Chilena de Neuropsiquiatría, volumen 41, No. 3 Santiago.

50. Rutter M, Andersen-Wood L, Beckett C, Bredenkamp D, Castle J, Groothues Ch, et al.(1999) Quasi-autistic patterns following severe early global privation. J Child Psychol Psychiat; 40: 537-549. 
51. Summerville MB, Kaslow NJ, Abbate MF and Cronan S.(1994) Psychopathology, family functioning, and cognitive style in urban adolescent with suicide attempts. J Abnorm Child Psychol; 22: 221-235.

52. Strozier AM and Armour MA.(1993) Family psychopathology: Cross training graduate marital and family therapy students in family assessment and psychiatric nosology. Contemporary Family Therapy; 15:33-49.

53. Shin LM, McNally RJ, Kosslyn SM, Thompson WL, Rauch SL Alpert NM, et al.(1999) Regional cerebral blood flow during script-driven imagery in childhood sexual abuse-related PTSD: a PET investigation. Am J Psychiatry; 156: $575-584$

54. Tomas J, Bargada M( 2002) Valoración de la familia en situación de crisis, disponible en http://www.latinsalud.com

55. Trenchi N. (2000) Impacto de la familia en el desarrollo de las personas, disponible en http://www.americasalud.com.uy/

56. Waughn C and Leff J.(1976) The influence of family and social factors on the course of psychiatric illness. Br J Psychiatry; 129: 125-137.

57. Weiss EL, Longhurst JG, Mazure CM.(1999) Childhood sexual abuse as a risk factor for depression in women: psychosocial and neurobiological correlates. Am J Psychiatry; 156: 816-828 [ $\underline{\text { Medline }] ~}$

58. Wright IK, Upton N, Marsden CA.(1991) Resocialization of isolation-reared rats does not alter their anxiogenic profile on the elevated X-maze model of anxiety. Physiol Behav; 50: 1129-1132

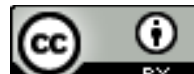

Este texto está protegido por una licencia Creative Commons 4.0.

Usted es libre para Compartir — copiar y redistribuir el material en cualquier medio o formato - y Adaptar el documen- to - remezclar, transformar y crear a partir del material- para cualquier propósito, incluso comercialmente, siempre que cumpla la condición de:

Atribución: Usted debe reconocer el crédito de una obra de manera adecuada, proporcionar un enlace a la licencia, e in- dicar si se han realizado cambios. Puede hacerlo en cualquier forma razonable, pero no de forma tal que sugiera que tie- ne el apoyo del licenciante o lo recibe por el uso que hace.
} 